\title{
Deposition of Polytetrafluoroethylene Film Assisted by Synchrotron Radiation Irradiation
}

\author{
Masaya Takeuchi $^{1^{*}}$, Hirokazu Izumi ${ }^{2}$, Mari Ishihara ${ }^{2}$, Toshiro Kobayashi ${ }^{3}$, \\ Akinobu Yamaguchi ${ }^{1}$, and Yuichi Utsumi ${ }^{1}$ \\ ${ }^{1}$ Laboratory and Advanced Science and Technology for Industry, University of Hyogo, \\ 3-1-2 Koto, Kamigori, Ako, Hyogo 678-1205, Japan \\ ${ }^{2}$ Hyogo Prefectural Institute of Technology, \\ 3-1-12 Yukihira, Suma, Kobe, Hyogo 645-0037, Japan \\ ${ }^{3}$ National Institute of Technology, Tsuyama College, \\ 624-1 Numa, Tsuyama, Okayama 708-8509, Japan \\ *masaya@lasti.u-hyogo.ac.jp
}

\begin{abstract}
A novel process was developed for fabricating a polytetrafluoroethylene (PTFE) thin film using synchrotron radiation (SR). First, a PTFE substrate was exposed to high-energy X-rays $(2-8 \mathrm{keV})$ at room temperature. Afterwards, the PTFE substrate (target) was heated under atmospheric pressure and fragments desorbed from the surface deposited on a glass substrate to produce a film with a thickness of above $10 \mu \mathrm{m}$. The characterization of the chemical structure of the deposited film was carried out using X-ray diffraction (XRD). The results indicated that the crystalline structure of the film became closer to those of the PTFE substrate upon an increase in the X-ray irradiation of the sample. The fabrication process of this PTFE thin film could be applied to various fields because surface modification of the substrate can be easily carried out.
\end{abstract}

Keywords: Synchrotron radiation, Polytetrafluoroethylene, Thin film, X-ray, Deposition

\section{Introduction}

Compared with other polymers, polytetrafluoroethylene (PTFE) has high heat resistance, excellent chemical stability, electrical insulation and low friction. Due to these outstanding properties, it is used in various applications, such as piping tube, packing in chemical plants, and cable coating. Also, thin films of PTFE are required for certain applications, such as proton exchange membranes [1] and membranes for desalination [2].

Several methods have been reported for fabricating thin films of PTFE, such as thermolysis [3], ion-beam sputtering [4], laser ablation [5-7], and synchrotron radiation (SR) [8-10]. These are performed by depositing fragments derived from PTFE on a substrate. Films deposited via SR can be specially fabricated with a high deposition rate, with compositions and crystalline features similar to those of the PTFE substrate. The method can be performed by irradiating a PTFE substrate with white light that has a photon energy of below $3 \mathrm{keV}$ to deposit fragments that adsorb on the surface of a Si (100) substrate. Meanwhile, the dry etching of PTFE using high-energy $(2-12 \mathrm{keV}) \mathrm{X}$-rays has also been studied [11-13]. X-rays are able to penetrate deeper into the PTFE substrate than photons with an energy below $3 \mathrm{keV}$, to induce chain scission of PTFE polymers. By utilizing the properties of high-energy X-rays, anisotropic pyrochemical etching has been proposed $[12,13]$, which is carried out by heating an X-ray irradiated PTFE substrate. An etching depth of $1 \mathrm{~mm}$ can be obtained using this method.

In this study, a novel process was developed for fabricating a PTFE thin film using SR. First, a PTFE substrate was subjected to high-energy X-rays (2$8 \mathrm{keV}$ ) at room temperature. Afterwards, the substrate (PTFE target) was heated under atmospheric pressure and fragments desorbed from the surface deposited on a glass substrate. As

Received March 29, 2019

Accepted April 15, 2019 
previously mentioned, high-energy X-ray photons can penetrate more deeply in the PTFE substrate to induce chain scission. Therefore, more fragments, which desorbed from the surface upon the heating of the PTFE target, are generated, significantly increasing the number of molecules that can form a deposited film. The chemical structure of the film was then evaluated using X-ray diffraction (XRD).

\section{Experimental}

PTFE thin films were prepared via the SR irradiation of PTFE substrates. The exposure was carried out on beamline BL-11 [14] of the NewSUBARU SR facility at the University of Hyogo, Japan. The X-ray beam was obtained from a bending magnet source inserted into a toroidal mirror, collimated by a mirror. The collimated beam was passed through two Be filters to exclude any photon energy below $2 \mathrm{keV}$ from reaching the exposure apparatus. The storage ring electron

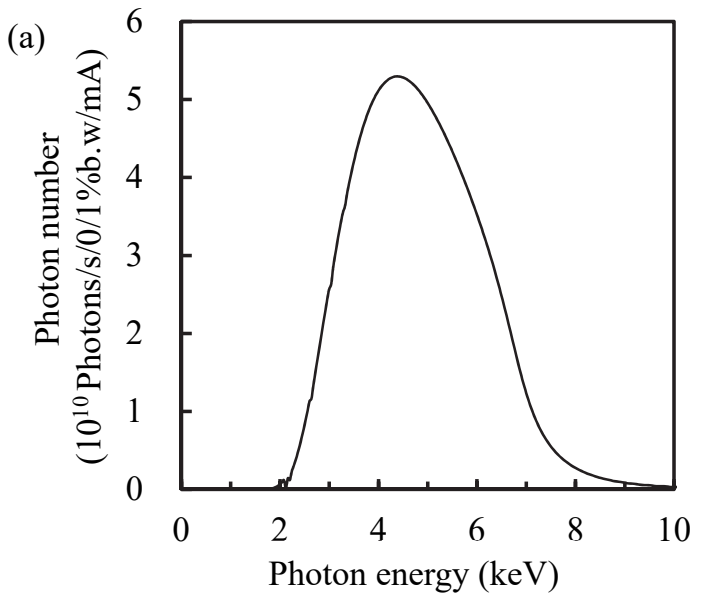

(b)

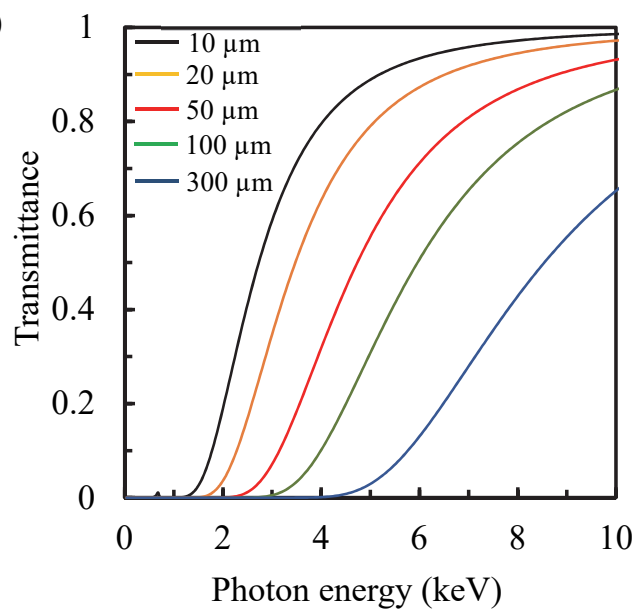

Fig. 1. (a) Calculated SR spectrum at $1.5 \mathrm{GeV}$ recorded at the exposure stage on beamline BL-11. (b) Photon energy dependence of X-ray transmittance through PTFE substrates with various thicknesses of 10, 20, 50, 100, and $300 \mu \mathrm{m}$. energy was set at $1.5 \mathrm{GeV}$. Figure 1 (a) shows the calculated spectrum of the X-ray beam at the exposure stage, which is continuous from $2-8 \mathrm{keV}$ with a peak photon energy of $4.5 \mathrm{keV}$. Figure 1 (b) shows the photon energy dependence of the transmittance through PTFE substrates with various thicknesses of $10,20,50,100$, and $300 \mu \mathrm{m}$. X-rays with higher photon energy are able to penetrate deeper into the film, causing scission of the main chain $\mathrm{C}-\mathrm{C}$ bonds, induced from the PTFE surface to a depth of around $300 \mu \mathrm{m}$ via X-ray irradiation on beamline BL- 11 .

A commercially available PTFE substrate (UNIVERSAL Co., Ltd., 00I-251-02) was used to prepare the PTFE target with a thickness of $1.5 \mathrm{~mm}$, which is enough to suppress the distortion of the substrate caused by heating during the deposition. The PTFE substrate was ultrasonically cleaned sequentially using acetone, 2-propanol, and pure water. The cleaned substrate was placed perpendicular to the incident X-ray beam in the exposure chamber of beamline BL-11 and vertically scanned at a scanning rate of $5 \mathrm{~mm} / \mathrm{s}$. The X-ray irradiation dose was controlled by changing the number of scans. Here helium $(\mathrm{He})$ gas was introduced into the exposure chamber to suppress the temperature rise of the PTFE substrate and evaporation of the depolymerized PTFE molecules during X-ray irradiation. The PTFE target was heated under atmospheric pressure on a hot plate for 1 hour and the fragments desorbed from the substrate surface were deposited onto a glass substrate (Matsunami Glass Ind., Ltd., C218181)

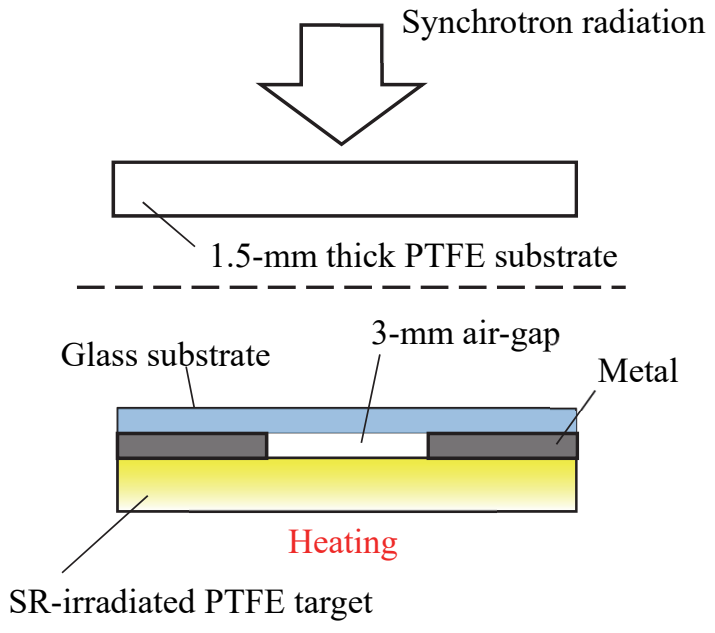

Fig. 2. Schematic diagram of thermal evaporation under atmospheric pressure of the SR-irradiated PTFE target. Deposition on a glass substrate was carried out by heating the PTFE target at $200{ }^{\circ} \mathrm{C}$. 
(Fig. 2). The temperature of the PTFE target was monitored an infrared camera (Nippon Avionics Co., Ltd., TVS-700) and the preset temperature of the hot plate was adjusted so that the substrate temperature was $200^{\circ} \mathrm{C}$. The air-gap distance between the PTFE target and the glass substrate was $3 \mathrm{~mm}$.

\section{Results and discussion}

The PTFE target was used to prepare deposited films using X-ray doses of $0.9,1.7,3.0$, and $4.3 \mathrm{~J} / \mathrm{mm}^{2}$. The deposited films were fabricated via heating of the PTFE target for 1 hour. The thicknesses of the deposited films were measured using a laser microscope (KEYENCE Co. Ltd., VK$8510)$ and were found to increase upon an increase in the X-ray doses of the PTFE targets (Fig. 3).

The crystalline structures of the deposited films on glass substrates were measured by XRD using a Rigaku-D/tex Ultra 250 equipped with a $\mathrm{CuK} \alpha$ radiation source $(\lambda=1.5406 \AA)$. The XRD spectrum of a $0.1 \mathrm{~mm}$ thick pristine PTFE substrate without SR irradiation is shown in Fig. 4 (a). Diffraction peaks derived from amorphous and crystalline material were observed at $2 \theta=16$ and $18^{\circ}$, respectively, which correspond with previously reported values $[9,12]$. Figure 4 (b) shows the XRD spectra of the deposited films fabricated using the PTFE target with various X-ray doses. Peaks were observed at $2 \theta=16$ and $18^{\circ}$ at $\mathrm{X}$-ray doses of 3.0 and $4.3 \mathrm{~J} / \mathrm{mm}^{2}$, similar to those observed for pristine PTFE. This result indicates that the crystalline structure of the deposited film is similar to that of PTFE at these X-ray doses.

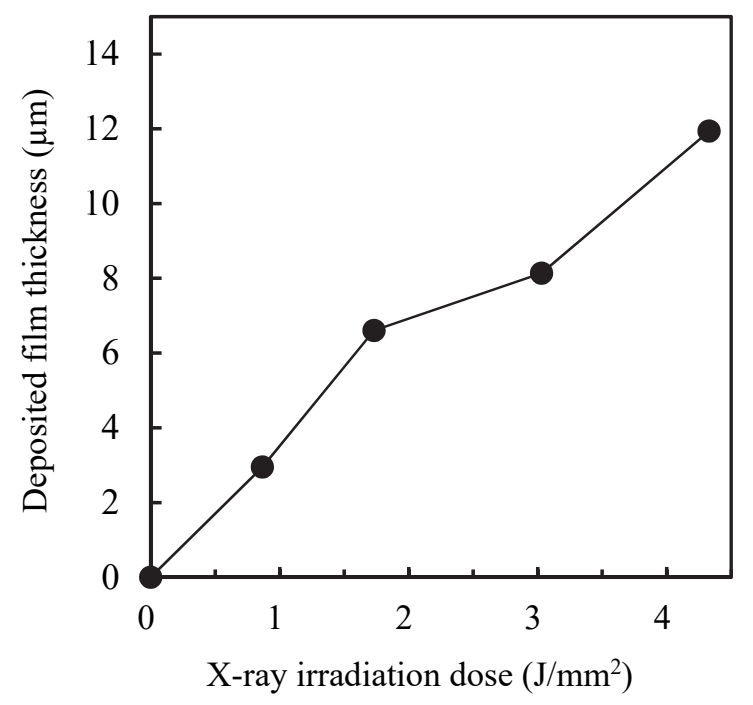

Fig. 3. The film thickness dependence on X-ray irradiation dose.
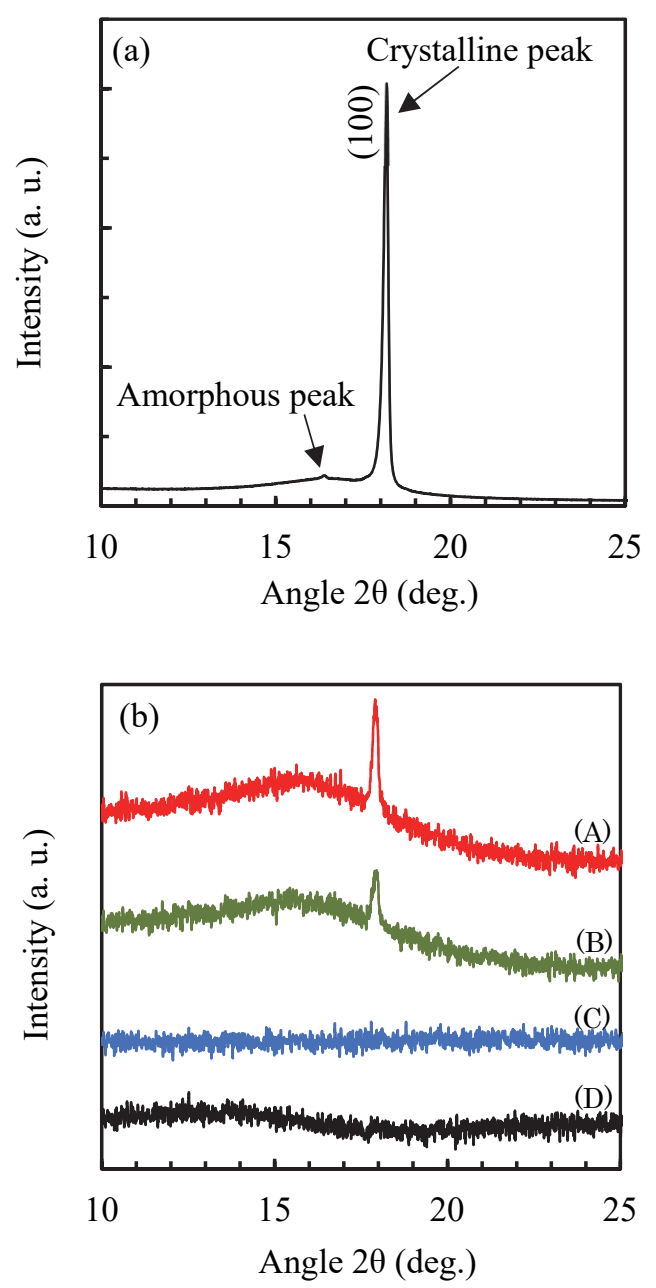

Fig. 4. XRD spectrum of (a) $0.1 \mathrm{~mm}$ thick PTFE and (b) the deposited film fabricated using the PTFE target with X-ray irradiation doses of (A) 4.3, (B) 3.0, (C) 1.7, and (D) $0.9 \mathrm{~J} / \mathrm{mm}^{2}$.

\section{Conclusion}

In this study, a novel deposition process was developed to fabricate a thin PTFE film using highenergy X-rays from $2-8 \mathrm{keV}$. A PTFE substrate was irradiated using $\mathrm{X}$-rays and the deposition was carried out under atmosphere pressure by heating the PTFE target and depositing the fragments desorbed from the surface onto a glass substrate. Using this method, a thin film with a thickness of above $10 \mu \mathrm{m}$ was achieved. The crystalline structures of the deposited films were evaluated using XRD. The XRD data of the samples were compared with those of the pristine PTFE substrate and it was found that the crystalline structures of the sample films are similar to those of the pristine PTFE substrate. The technique used in this study to fabricate PTFE thin films can be easily used to perform surface modification and can be applied to various applications. 


\section{References}

1. F. Liu, B. Yi, D. Xing, J. Yu, and H. Zhang, $J$. Memb. Sci., 212 (2003) 213.

2. H. Zhu, H. Wang, F. Wang, Y. Guo, H. Zhang, and J. Chen, J. Memb. Sci., 446 (2013) 145.

3. T. C. Nason, J. A. Moore, and T.-M. Lu, Appl. Phys. Lett., 60 (1992) 1866.

4. F. Quaranta and A. Valentini, Appl. Phys. Lett., 63 (1993) 10.

5. S. G. Hansen and T. E. Robitaille, Appl. Phys. Lett., 52 (1988) 81.

6. G. B. Blanchet and S. I. Shah, Appl. Phys. Lett., 62 (1993) 1026.

7. Y. Ueno, T. Fuji, and F. Kannari, Appl. Phys. Lett., 65 (1994) 1370.

8. M. Inayoshi, M. Ikeda, M. Hori, T. Goto, M.
Hiramatsu, and A. Hiraya, Jpn. J. Appl. Phys., 34 (1995) L1675.

9. T. Katoh and Y. Zhang, Appl. Phys. Lett., 68 (1996) 865.

10. M. Inayoshi, M. Ito, M. Hori, and T. Goto, $J$. Vac. Sci. Technol. B, 17 (1999) 949.

11. Y. Ukita, M. Kishihara, K. Kanda, S. Matsui, K. Mochiji, and Y. Utsumi, Jpn. J. Appl. Phys., 47 (2008) 337.

12. A. Yamaguchi, H. Kido, Y. Ukita, M. Kishihara, and Y. Utsumi, Appl. Phys. Lett., 108 (2016) 051610 .

13. A. Yamaguchi, H. Kido, and Y. Utsumi, $J$. Photopolym. Sci. Technol., 29 (2016) 403.

14. M. Takeuchi, A. Yamaguchi, and Y. Utsumi, $J$. Synchrotron. Rad., 26 (2019) 528. 